\title{
APLICAÇÃO DE ENSAIOS ELÉTRICOS E ELETROMAGNÉTICOS \\ EM PROBLEMA DE POLUIÇÃO AMBIENTAL
}

Jorge Eduardo da Silva, ${ }^{1}$ Elaine Maria Lopes Loureiro, ${ }^{2}$ Vagner Roberto Elis, ${ }^{3}$ Francisco Yukio Hiodo, ${ }^{4}$ Jorge Luís Porsani ${ }^{5}$

Recebido em 8 ago., 2002 / Aceito em 17 dez., 2003

Received Aug. 8, 2002 / Accepted Dec. 17, 2003

\section{ABSTRACT}

This work aims at the detection of the contamination caused by a waste disposal area, through the electric geophysical methods of resistivity, induced electromagnetics. Field measurements had been carried out in Ribeirão Preto, São Paulo, Brazil, in a deactivated waste disposal area. The obtained data had been dealt with appropriate soffwares that enabled the distinction of the anomalies which help the characterization of the environmental pollution problem.

Keywords: Electric Resistivity, Electromagnetic Induction, Landfill, Ribeirão Preto, São Paulo, Brazil.

\section{RESUMO}

Este trabalho visa o estudo de contaminação causada por um lixão, através dos métodos geofíisicos da resistividade, eletromagnético indutivo. Os trabalhos foram realizados em Ribeirão Preto, São Paulo, Brasil, em um lixão desativado. Os dados obtidos foram tratados com soffwares apropriados que possibilitaram o realce das anomalias, as quais auxiliaram na caracterização do problema ambiental causado.

Palavras-chave: Resistividade elétrica, Indução eletromagnética, Contaminação ambiental, lixão de Ribeirão Preto.

\footnotetext{
Graduação em Geofísica-IAG/USP - Rua do Matão, 1226 - Cidade Universitária - São Paulo-SP - CEP: 05508-090 - Tel.: ( 11 ) 3091-4775 - Fax: (11) 3091-5034 - E-mail: quartafeira@ @hotmail.com

2 Graduação em Geofísica-IAG/USP - Rua do Matão, 1226 - Cidade Universitária - São Paulo-SP - CEP.: 05508-090 - Telefone: (0xx11) 3091-4775 - Fax: (0xx11) 3091-5034 - E-mail: Não tem

31 IAG-USP - Departamento de Geofísica - Rua do Matão 1226 - Cidade Universitária - São Paulo - SP - CEP: $05508-090$ - Tel.: (11) 30914734 - Fax: (11) 30915034 - E-mail: vagnelis@iag.usp.br

4 IAG-USP - Departamento de Geofísica - Rua do Matão 1226 - Cidade Universitária - São Paulo - SP - CEP: 05508 -090 - Tel.: (11) 30914777 - Fax: (11) 30915034 - E-mail: francisc@iag.usp.br

5 IAG-USP - Departamento de Geofísica - Rua do Matão 1226 - Cidade Universitária - São Paulo - SP - CEP: 05508-090 - Tel.: (11) 30914734 - Fax: (11) 30915034 - E-mail: porsani@iug.usp.br
} 


\section{INTRODUÇÃOO}

Exemplos de aplicação de geofísica no estudo da contaminação gerada por resíduos urbanos podem ser encontrados na literatura desde o final da década de 1960 e início dos anos 70, sendo o método da eletrorresistividade amplamente utilizado (STOLLAR; ROUX, 1975; KELLY, 1976). Elis \& Zuquette (1997) e Elis (1999) mostraram a possibilidade de aplicação dos metódos da eletrorresistividade, com o uso de sondagens elétricas verticais e caminhamento elétrico dipólo-dipólo no estudo de áreas utilizadas para disposição de resíduos urbanos e industriais. Foram obtidas informações importantes, como profundidade da zona saturada, fluxo subterrâneo, posição dos resíduos, formato e extensão da pluma de contaminação. Neste trabalho foram utilizados os métodos elétricos da resistividade para detecção da pluma de contaminação originada por um lixão desativado. A área estudada, na região de Ribeirão Preto, situada na porção nordeste do estado de São Paulo, Brasil, latitude $21^{\circ} 10^{\prime}$, longitude $47^{\circ} 48^{\prime}$. Os dados obtidos foram tratados com soffwares apropriados que possibilitaram o realce das anomalias relacionadas às zonas com problemas de contaminação.

\section{CARACTERIZAÇÃO DA ÁREA DE ESTUDO}

A área caracteriza-se como um divisor de águas superficiais que tem como substrato rochoso os arenitos não-silicificados da Formação Botucatu, como pode ser observado na Figura 1. Esse material é recoberto por materiais inconsolidados constituídos na parte inferior por solos residuais dos arenitos e na parte superior por materiais argilosos retrabalhados e oriundos das áreas mais elevadas topograficamente constituídas pelos magmatitos (basaltos) da Formação Serra Geral (ZUQUETTE; GANDOLFI; PEJON, 1990). No local de estudo através dos métodos geofísicos foram dispostos aproximadamente $600.000 \mathrm{~m}^{3} \mathrm{de}$ resíduos sólidos domésticos em duas cavas alongadas com profundidade média de $10 \mathrm{~m}$ (Figura 2).
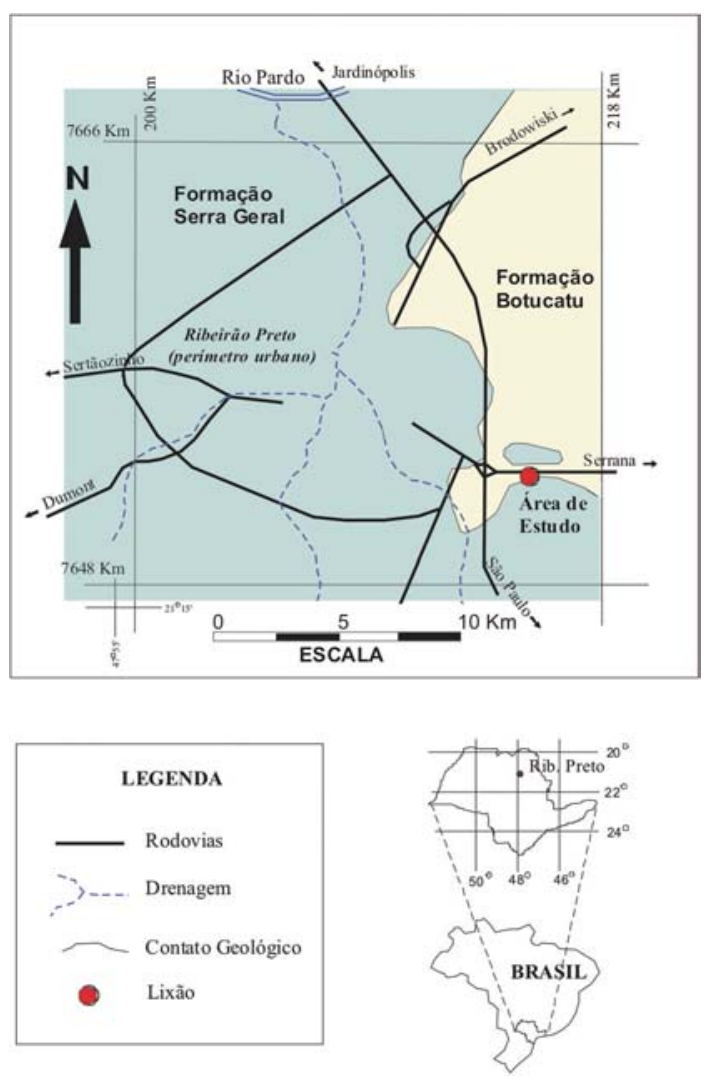

Figura 1 - Localização da área de pesquisa.

Figure 1- Location of research area. 


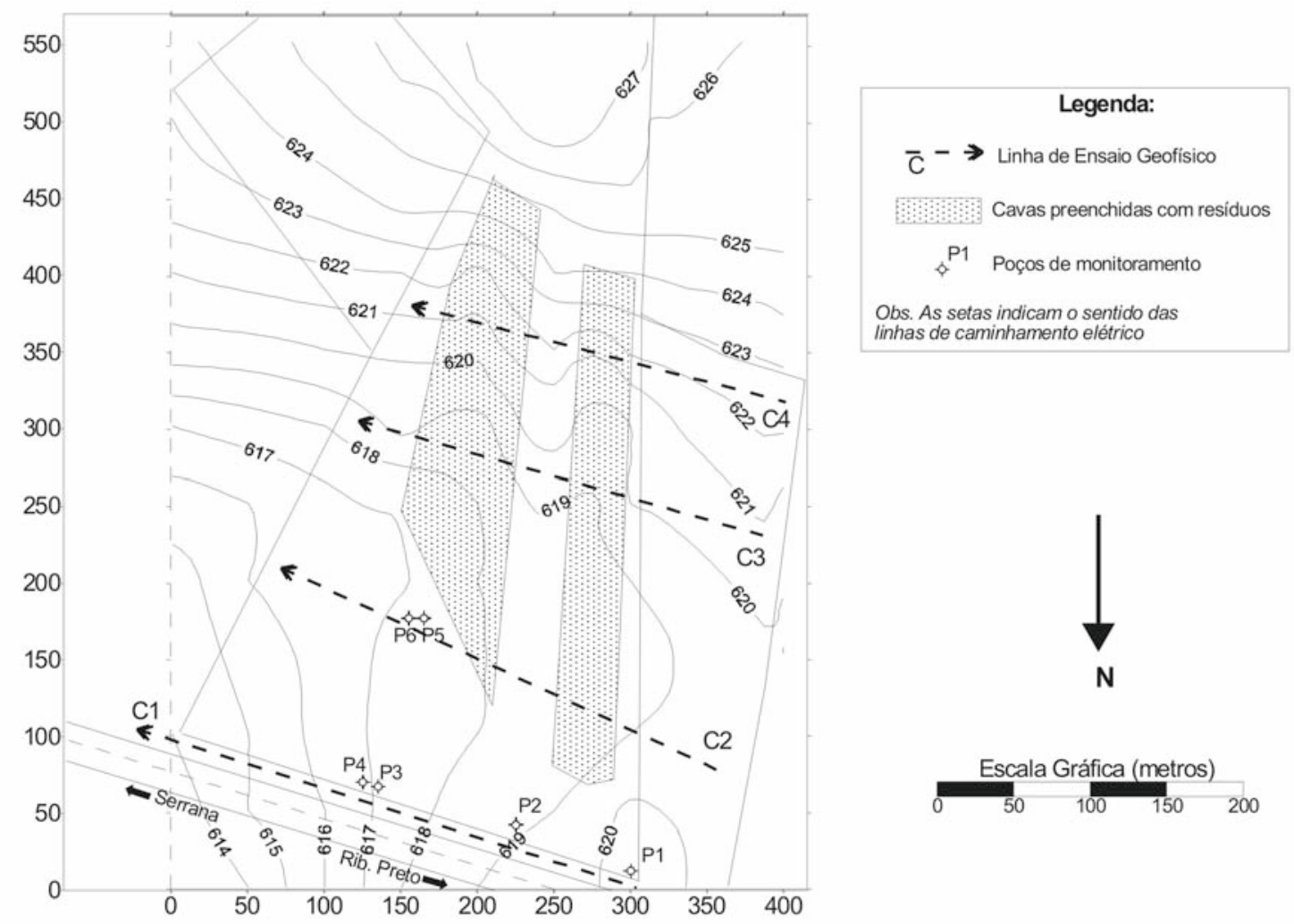

Figura 2 - Localização das linhas de ensaio

Figure 2- Location of tests profiles.

\section{METODOLOGIA}

0 método da eletrorresistividade emprega uma corrente elétrica artificial que é introduzida no terreno através de dois eletrodos (denominados de A e B), com o objetivo de medir o potencial gerado em outros dois eletrodos (denominados de $\mathrm{M}$ e N) nas proximidades do fluxo de corrente, permitindo assim calcular a resistividade real ou aparente em subsuperfície. A resistividade (e seu inverso, a condutividade) de solos e rochas sofre a influência da quantidade de água contida e da salinidade dessa água, o que permite uma imensa possibilidade de aplicação do método em estudos ambientais e hidrogeólogicos, sendo a presença de água e de substâncias contaminantes facilmente detectadas. A variedade de técnicas de aplicação de ensaios (caminhamentos e sondagens) confere ao método uma versatilidade muito grande, que possibilita, no caso específico de áreas de disposição de resíduos, a aplicação de técnicas e arranjos diferentes que atendem às necessidades de investigação dos mais variados parâmetros importantes para a caracterização da área e determinação da zona contaminada. A técnica utilizada neste trabalho foi o caminhamento dipólo-dipólo com espaçamento de $10 \mathrm{~m}$ que possibilitou a observação da variação de resistividade aparente em cinco níveis teóricos.

No método eletromagnético indutivo foram utilizadas duas bobinas: uma transmissora Tx e outra receptora Rx. Acoplada a bobina transmissora existe um módulo de controle e emissão de corrente elétrica, alimentado por uma bateria. À bobina receptora está acoplado um módulo de leitura que fornece valores de condutividade aparente do terreno. Durante a operação, é injetada uma corrente alternada na bobina transmissora, que vai gerar um campo magnético primário Hp, ortogonal à disposição da bobina. Esse campo magnético primário vai induzir correntes elétricas no subsolo, sendo assim energizado. Passando por um condutor, essas correntes vão dar origem a um campo magnético 
secundário Hs, que será lido pela bobina receptora, juntamente com 0 campo magnético primário. A razão entre Hs e Hp é usada pelo equipamento para medir diretamente a condutividade aparente.

As bobinas podem ser dispostas em duas configurações: coplanar horizontal e vertical, o que altera a profundidade teórica de investigação.

Na área de estudo foram realizadas quatro linhas de caminhamento elétrico e eletromagnético (Linhas $\mathrm{Cl}$ a (4). As linhas C2 a C4 estão posicionadas sobre as cavas e a Linha Cl está posicionada a norte das cavas, a jusante do fluxo subterrâneo local. Essa disposição dos ensaios possibilita avaliar se a contaminação está restrita às cavas ou se está ocorrendo migração de contaminantes dentro da zona saturada.. Os dados dos ensaios elétricos e eletromagnéticos foram apresentados utilizando o software Surfer 7.0 da Golden Software, Inc.

\section{RESULTADOS}

Os ensaios realizados permitiram elaborar pseudo-seções de resistividade e condutividade aparente com vários níveis de investigação, possibilitando a observação de zonas anômalas. Tais anomalias são relacionadas a presença de resíduos e líquidos poluentes das cavas e da infiltração de polventes (chorume) no solo que penetram na zona saturada sendo dispersos pelo fluxo de água. As pseudo-seções de resistividade aparente e condutividade aparente da Linha $\mathrm{Cl}$ (figuras 3 e 4) ilustram bem esta última situação, pois os ensaios foram feitos fora das cavas do lixão, mas no entanto apresentam anomalia de baixa resistividade e alta condutividade entre $120-80 \mathrm{~m}$ entre as profundidades teóricas de 20 e 30m, indicando que a contaminação está avançando

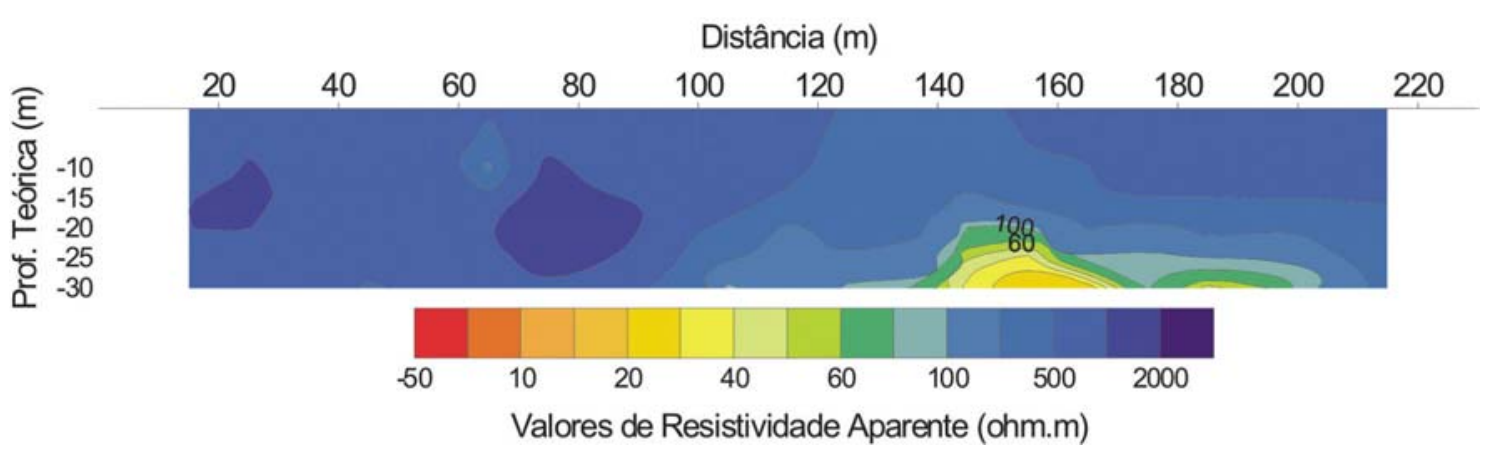

Figura 3 - Pseudo-seção de resistividade aparente - Linha Cl Figure 3-Pseudo section of apparent resistivity-Line Cl.

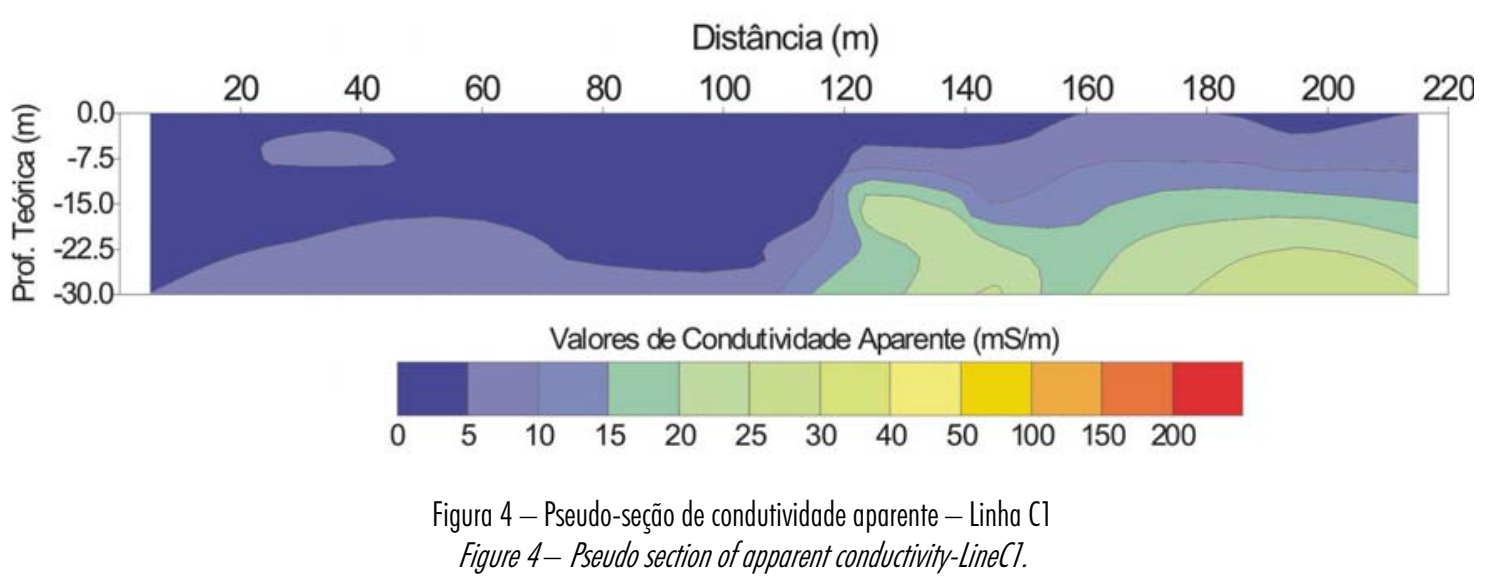


dentro da zona saturada e para fora da área do lixão. As pseudo-seções da Linha C4 (Figuras 5 e 6) foram realizadas cortando as cavas e apresentam zonas de baixa resistividade e alta condutividade entre 80 e $220 \mathrm{~m}$, da superfície até a profundidade teórica de $30 \mathrm{~m}$. Essas anomalias condutivas representam as cavas preenchidas com resíduos $e$ chorume.
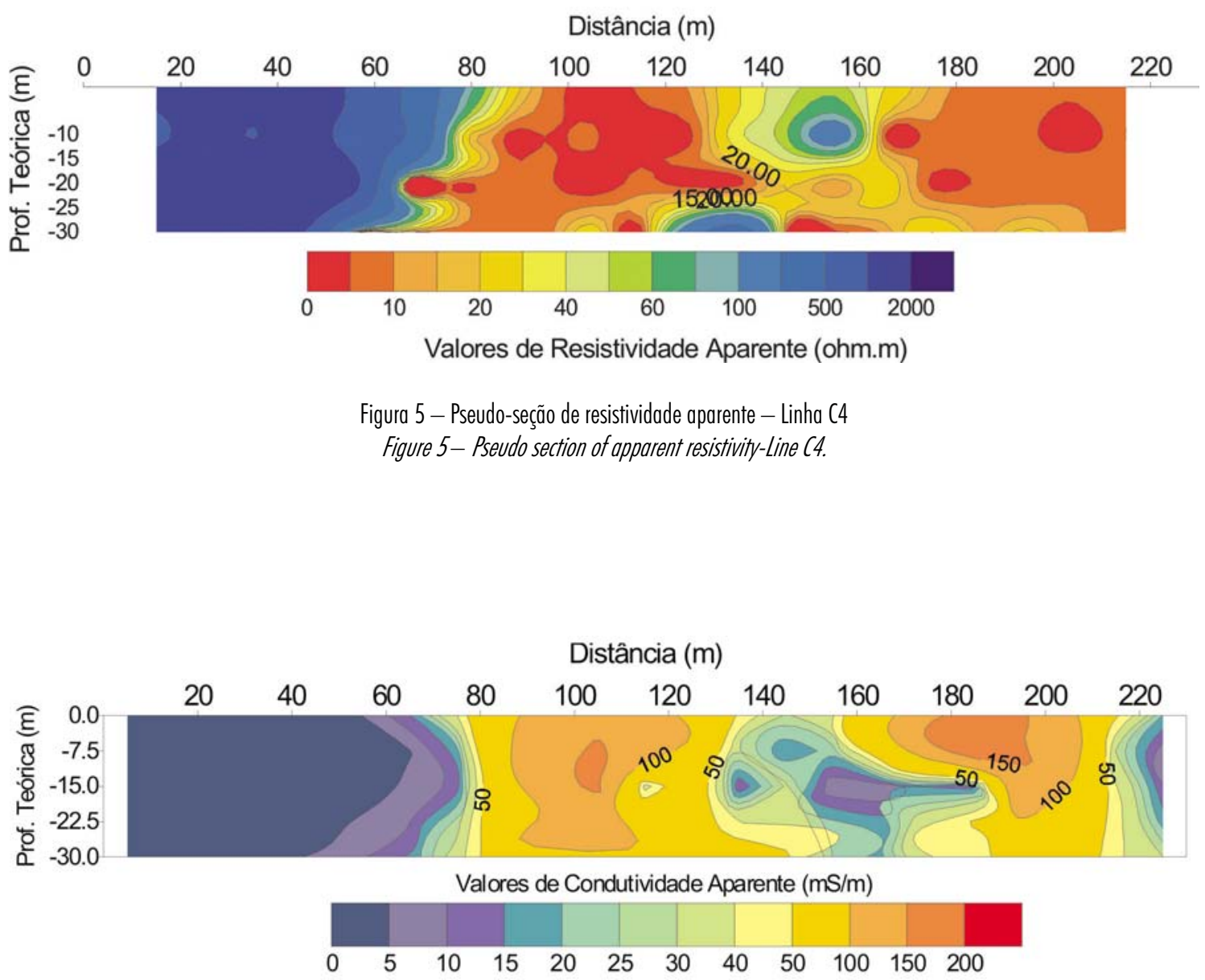

Figura 6 - Pseudo-seção de condutividade aparente - Linha C4 Figure 6-Pseudo section of apparent conductivity-Line C4. 


\section{REFERÊNCIAS}

ELIS, V. R.; ZUQUETTE, L.V. Determinação da estrutura do aterro sanitário de Ribeirão Preto, São Paulo, através de métodos geoelétricos. In: CONGRESSO INTERNACIONAL DA SOCIEDADE BRASILEIRA DE GEOFÍSICA, 5., 1997, São Paulo. Anais... São Paulo: Sociedade Brasileira de Geofísica, 1997. p. 417-420.

Avaliação da aplicabilidade de métodos elétricos de prospecção geofísica no estudo de áreas utilizadas para disposição de resíduos. Tese (Doutorado)-Instituto de Geociências e Ciencias Exatas, Universidade Estadual de São Paulo, Rio Claro, 1999. Trabalho inédito.
KELLY, W. E. Geoelectric sounding for delineating groundwater contamination. Ground Water, [S.I.], v. 14, n. 1, p. 6-10, 1976.

STOLLAR, R. L.; ROUX, P. Earth resistivity surveys: a method for delineating groundwater contamination. Ground Water, [S.I.], v. 13, n. 2, p. 145150, 1975.

ZUQUETTE, L.V.; GANDOLFI, N.; PEJON, 0. J. 0 mapeamento geotécnico na previsão e prevenção de riscos geológicos em áreas urbanas. In: SIMPÓSIO LATINO AMERICANO SOBRE RISCO GEOLÓGICO URBANO, 1. 1990, São Paulo. Atas... São Paulo: Associação Brasileira de Geologia de Engenharia, 1990. p. 305-315, 1990.

\section{NOTAS SOBRE OS AUTORES}

Jorge Eduardo da Silva é bacharel em Geofísica pelo Instituto de Astronomia, Geofísica e de Ciências Atmosféricas da Universidade de São Paulo (IAG - USP) em 2003. Atualmente trabalha como Geofísico na área de aquisição, processamento e interpretação de dados geofísicos junto à empresa AeroGeoPhysica Latinoamerica (AGP - LA).

Elaine Maria Lopes Loureiro é bacharel em Geofísica pelo Instituto de Astronomia, Geofísica e de Ciências Atmosféricas da Universidade de São Paulo (IAG - USP) em 2003. Atualmente trabalha como Geofísica à empresa IDS Radar.

Vagner Roberto Elis é graduado em Geologia (1990) pela Universidade Estadual Paulista. Mestre em Geociências pela Universidade Estadual Paulista (1993), desenvolvendo a dissertação "A aplicação da geofísica para análise do meio físico: importância para elaboração de mapeamento geotécnico". Doutor em Geociências pela Universidade Estadual Paulista (1999), defendendo a tese "Avaliação da aplicabilidade de métodos elétricos de prospecçãa geofísica no estudo de áreas utilizadas para disposição de resíduos". Docente do Departamento de Geofísica do Instituto de Astronomia, Geofísica e Ciências Atmosféricas da Universidade de São Paulo, com atuação nas áreas de Geofísica Aplicada a estudos ambientais e hidrogeológicos.

Francisco Yukio Hiodo é graduado em Física pelo Instituto de Física da Universidade de São Paulo-USP (1972). Mestrado em Geofísica pelo Instituto de Astronomia, Geofísica e Ciências Atmostéricas (IAG-USP) (1981). Doutorado em Geofísica pelo IAG-USP (1990). Auxiliar de Ensino no IAG-USP (1973). Professor assistente em 1981. Professor Doutor do IAG-USP desde 1990. Atualmente é especialista em Instrumentação Geofísica nas áreas de Paleomagnetísmo (magnetômetros rotativos e desmagnetizadores), Geofísica Nuclear (espectrômetros gama e emanômetros de radônio), Perfilagem de poços tubulares, Geotermometria e sensores de temperatura, Magnetômetros de precessão de prótons e fluxgate, Métodos geoelétricos e eletromagnéticos.

Jorge Luís Porsani é geólogo pelo Instituto de Geociências da UFBa (1987). Mestrado em Geofísica pelo Núcleo de Pesquisas Geofísicas Aplicadas à Prospecção de Hidrocarbonetos da UFPa (1991). De 1991 a 1996, trabalhou como Geofísico no Centro de Pesquisas da PETROBRAS. Doutorado em Geociências e Meio Ambiente pelo Instituto de Geociências e Ciências Exatas da UNESP (1999). Desde 1998 é docente do Departamento de Geofísica do IAG/USP, atuando com métodos geoelétricos aplicados à geologia, geotecnia, meio ambiente e arqueologia. Atualmente, é vice-coordenador do Programa de Pós-Graduação em Geofísica e coordenador do Projeto de Instalaçãa do Sítio Controlado de Geofísica Rasa do IAG (Fapesp 02/07509-1). 